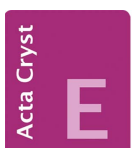

CRYSTALLOGRAPHIC COMMUNICATIONS

ISSN 2056-9890

Received 3 October 2016

Accepted 4 October 2016

Edited by W. T. A. Harrison, University of Aberdeen, Scotland

Keywords: crystal structure; strontium tellurite; channel structure; stereoactive electron lone pair.

CCDC reference: 1508062

Supporting information: this article has supporting information at journals.iucr.org/e

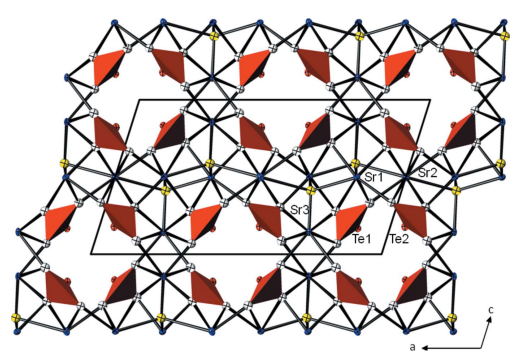

OPEN $\odot$ ACCESS

\section{Crystal structure of $\operatorname{Sr}_{5} \mathrm{Te}_{4} \mathrm{O}_{12}(\mathrm{OH})_{2}$, the first basic strontium oxotellurate(IV)}

\author{
Matthias Weil* and Mahdi Shirkhanlou
}

Institute for Chemical Technologies and Analytics, Division of Structural Chemistry, Vienna University of Technology, Getreidemarkt 9/164-SC, A-1060 Vienna, Austria. *Correspondence e-mail: matthias.weil@tuwien.ac.at

The asymmetric unit of the title basic strontium oxotellurate(IV), $\mathrm{Sr}_{5} \mathrm{Te}_{4} \mathrm{O}_{12}(\mathrm{OH})_{2}$ \{systematic name pentastrontium tetrakis[oxotellurate(IV)] dihydroxide\}, comprises three $\mathrm{Sr}^{\mathrm{II}}$ cations (one with site symmetry 2) and two $\mathrm{Te}^{\mathrm{IV}}$ atoms, as well as seven $\mathrm{O}$ atoms. The coordination numbers of the alkaline earth cations to nearby $\mathrm{O}$ atoms range from seven $(2 \times)$ to eight, and the $\mathrm{Te}^{\mathrm{IV}}$ atoms are surrounded by three oxygen partners in the form of trigonal pyramids. The $\mathrm{SrO}_{x}$ polyhedra share corners and edges to build up a three-dimensional framework structure encapsulating channels propagating along [010]. The $\mathrm{Te}^{\text {IV }}$ atoms flank the framework $\mathrm{O}$ atoms and are situated at the outer array of the channels with the $5 s^{2}$ lone electron pairs protruding into the empty space of the channels (diameter $\simeq 4 \AA$ ). Although the $\mathrm{H}$ atom of the $\mathrm{OH}$ group could not be located, bond-valence-sum calculations and typical $\mathrm{O} \cdots \mathrm{O}$ distances (range 2.81-3.06 $\AA$ ) clearly indicate hydrogen bonding of medium to weak strengths.

\section{Chemical context}

The peculiar feature of the crystal chemistry of oxotellurates(IV) (Christy et al., 2016) is the presence of the $5 s^{2}$ lone electron pair, denoted $E$. In the majority of cases, the lone electron pair $E$ is stereochemically active, making oxotellurates(IV) interesting for crystal engineering, e.g. in terms of the synthesis of compounds with non-centrosymmetric structures or structures with polar directions. Next to the influence of the (metal) cation on the physico-chemical characteristics of oxotellurates(IV), physical and underlying structural properties of such compounds can also be varied by incorporation of other oxoanions into the oxotellurate(IV) framework, e.g. by p-block oxoanions such as nitrate (Stöger \& Weil, 2013) or selenate (Weil \& Shirkanlou, 2015), or by $d$-block oxoanions such as vanadate (Weil, 2015).

In this context we attempted the hydrothermal synthesis of new oxotellurate phases in the system $\mathrm{Sr}-\mathrm{Te}-\mathrm{Se}-\mathrm{O}-(\mathrm{H})$. In comparison with typical solid-state reactions using open crucibles under atmospheric conditions, this method is more feasible because $\mathrm{Te}^{\mathrm{IV}}$ then tends not to be oxidized or to be evaporated during the reaction process. However, a clear disadvantage of the hydrothermal method is the high(er) number of adjustable parameters (pressure, concentration, temperature, time, filling degree, solvent etc), which often makes the products of these experiments difficult to predict or even to reproduce, accompanied by formation of several solid phases in one batch. This was also the case for the present study. Instead of a strontium oxoselenatotellurate, several oxotellurate phases were obtained without incorporation of selenium. Amongst these phases, the title compound, 
Table 1

Selected geometric parameters $\left(\AA,^{\circ}\right)$.

\begin{tabular}{|c|c|c|c|}
\hline $\mathrm{Sr} 1-\mathrm{O} 7$ & $2.430(12)$ & $\mathrm{Sr} 3-\mathrm{O} 2$ & $2.507(11$ \\
\hline $\mathrm{Sr} 1-\mathrm{O}^{\mathrm{i}}$ & $2.476(12)$ & $\mathrm{Sr} 3-\mathrm{O} 4^{\mathrm{v}}$ & $2.517(11)$ \\
\hline $\mathrm{Sr} 1-\mathrm{O} 1^{\mathrm{ii}}$ & $2.593(12)$ & $\mathrm{Sr} 3-\mathrm{O}^{\mathrm{i}}$ & $2.536(11)$ \\
\hline $\mathrm{Sr} 1-\mathrm{O} 3$ & $2.596(11)$ & $\mathrm{Sr} 3-\mathrm{O}^{\mathrm{vi}}$ & $2.590(12)$ \\
\hline $\mathrm{Sr} 1-\mathrm{O} 2^{\mathrm{iii}}$ & $2.616(12)$ & $\mathrm{Sr} 3-\mathrm{O} 4^{\mathrm{vii}}$ & $2.644(11)$ \\
\hline $\mathrm{Sr} 1-\mathrm{O}^{\mathrm{iii}}$ & $2.700(11)$ & $\mathrm{Sr} 3-\mathrm{O} 1^{\text {viii }}$ & $2.666(11)$ \\
\hline $\mathrm{Sr} 1-\mathrm{O} 2$ & $2.852(12)$ & $\mathrm{Te} 1-\mathrm{O} 6$ & $1.865(11)$ \\
\hline $\mathrm{Sr} 2-\mathrm{O} 3$ & $2.510(11)$ & $\mathrm{Te} 1-\mathrm{O} 2$ & $1.871(11)$ \\
\hline $\mathrm{Sr} 2-\mathrm{O} 1^{\mathrm{iv}}$ & $2.624(12)$ & $\mathrm{Te} 1-\mathrm{O} 5$ & $1.890(12$ \\
\hline $\mathrm{Sr} 2-\mathrm{O} 5$ & $2.633(12)$ & $\mathrm{Te} 2-\mathrm{O} 4$ & $1.858(11$ \\
\hline $\mathrm{Sr} 2-\mathrm{O} 7$ & $2.960(11)$ & $\mathrm{Te} 2-\mathrm{O} 3$ & $1.882(11)$ \\
\hline $\mathrm{Sr} 3-\mathrm{O} 7^{\mathrm{iii}}$ & $2.393(11)$ & $\mathrm{Te} 2-\mathrm{O} 1$ & $1.886(11$ \\
\hline $\mathrm{O} 6-\mathrm{Te} 1-\mathrm{O} 2$ & $99.4(4)$ & $\mathrm{O} 4-\mathrm{Te} 2-\mathrm{O} 3$ & $101.1(5)$ \\
\hline $\mathrm{O} 6-\mathrm{Te} 1-\mathrm{O} 5$ & $100.3(5)$ & $\mathrm{O} 4-\mathrm{Te} 2-\mathrm{O} 1$ & $100.3(5)$ \\
\hline $\mathrm{O} 2-\mathrm{Te} 1-\mathrm{O} 5$ & $98.0(5)$ & $\mathrm{O} 3-\mathrm{Te} 2-\mathrm{O} 1$ & $98.8(5)$ \\
\hline
\end{tabular}

Symmetry codes: (i) $x, y-1, z$; (ii) $-x, y,-z+1$; (iii) $-x+\frac{1}{2}, y-\frac{1}{2},-z+1$; (iv) $-x, y+1,-z+1$; (v) $x+\frac{1}{2}, y-\frac{1}{2}, z$; (vi) $-x+\frac{1}{2}, y-\frac{1}{2},-z$; (vii) $-x, y,-z$; (viii) $x+\frac{1}{2}, y+\frac{1}{2}, z$.

$\mathrm{Sr}_{5} \mathrm{Te}_{4} \mathrm{O}_{12}(\mathrm{OH})_{2}$, a hitherto unknown strontium oxotellurate, was isolated and structurally determined by single crystal $\mathrm{X}$-ray diffraction.

\section{Structural commentary}

The asymmetric unit of $\mathrm{Sr}_{5} \mathrm{Te}_{4} \mathrm{O}_{12}(\mathrm{OH})_{2}$ comprises three $\mathrm{Sr}$, two Te and seven $\mathrm{O}$ atoms ( $\mathrm{H}$ atoms were not included in the final model, see Section 5 and discussion below). Except one $\mathrm{Sr}$ atom $(\mathrm{Sr} 2)$ that is located on a twofold rotation axis, all atoms are in general positions.

The coordination numbers of the $\mathrm{Sr}$ atoms are 7 (for $\mathrm{Sr} 1$ and $\mathrm{Sr} 3$ ) and 8 (for $\mathrm{Sr} 2$ ) if $\mathrm{Sr}-\mathrm{O}$ distances $<3.0 \AA$ are considered as relevant for the first coordination sphere. The
Table 2

Results of the bond-valance-sum (BVS) analysis.

\begin{tabular}{lll}
\hline Atom & BVS & $\Delta$ to expected value \\
\hline $\mathrm{Sr} 1$ & 2.07 & 0.07 \\
$\mathrm{Sr} 2$ & 1.91 & 0.09 \\
$\mathrm{Sr} 3$ & 2.23 & 0.23 \\
$\mathrm{Te} 1$ & 3.94 & 0.06 \\
$\mathrm{Te} 2$ & 3.93 & 0.07 \\
$\mathrm{O} 1$ & 2.04 & 0.04 \\
$\mathrm{O} 2$ & 2.08 & 0.08 \\
$\mathrm{O} 3$ & 1.91 & 0.09 \\
$\mathrm{O} 4$ & 1.96 & 0.04 \\
$\mathrm{O} 5$ & 1.89 & 0.11 \\
$\mathrm{O} 6$ & 1.95 & 0.05 \\
$\mathrm{O} 7$ & 1.21 & 0.79
\end{tabular}

BVS parameters of Brown \& Altermatt (1985) were used for all bonds.

corresponding polyhedra are considerably distorted, with $\mathrm{Sr}-$ O bond lengths ranging from 2.393 (11) to 2.960 (11) $\AA$ (Table 1) and might be described as monocapped octahedra for $\mathrm{Sr} 1$ and $\mathrm{Sr} 3$, and as a bicapped trigonal prism for $\mathrm{Sr} 2$. The $\mathrm{SrO}_{8}$ and the two $\mathrm{SrO}_{7}$ polyhedra share corners and edges, thereby constructing a three-dimensional framework structure encapsulating channels that propagate along [010]. Each of the two Te atoms connect to the outer oxygen atoms of the framework in a very similar trigonal-prismatic configuration (Table 1), with the $5 s^{2}$ lone electron pair $E$ being stereochemically active, i.e. pointing towards the empty space of the channels (Fig. 1). The channel diameter (without contribution of the lone pairs) is $\simeq 4 \AA$. Te-O bond lengths [1.865 (11)1.890 (12) $\AA$ for Te1 and 1.858 (11)-1.886 (11) $\AA$ for Te2] and $\mathrm{O}-\mathrm{Te}-\mathrm{O}$ angles $\left[98.0(5)-100.3(5)^{\circ}\right.$ for $\mathrm{Te} 1$ and $98.8(5)-$ $101.1(5)^{\circ}$ for Te2] are typical for oxotellurate(IV) anions with three oxygen partners (Christy et al., 2016).

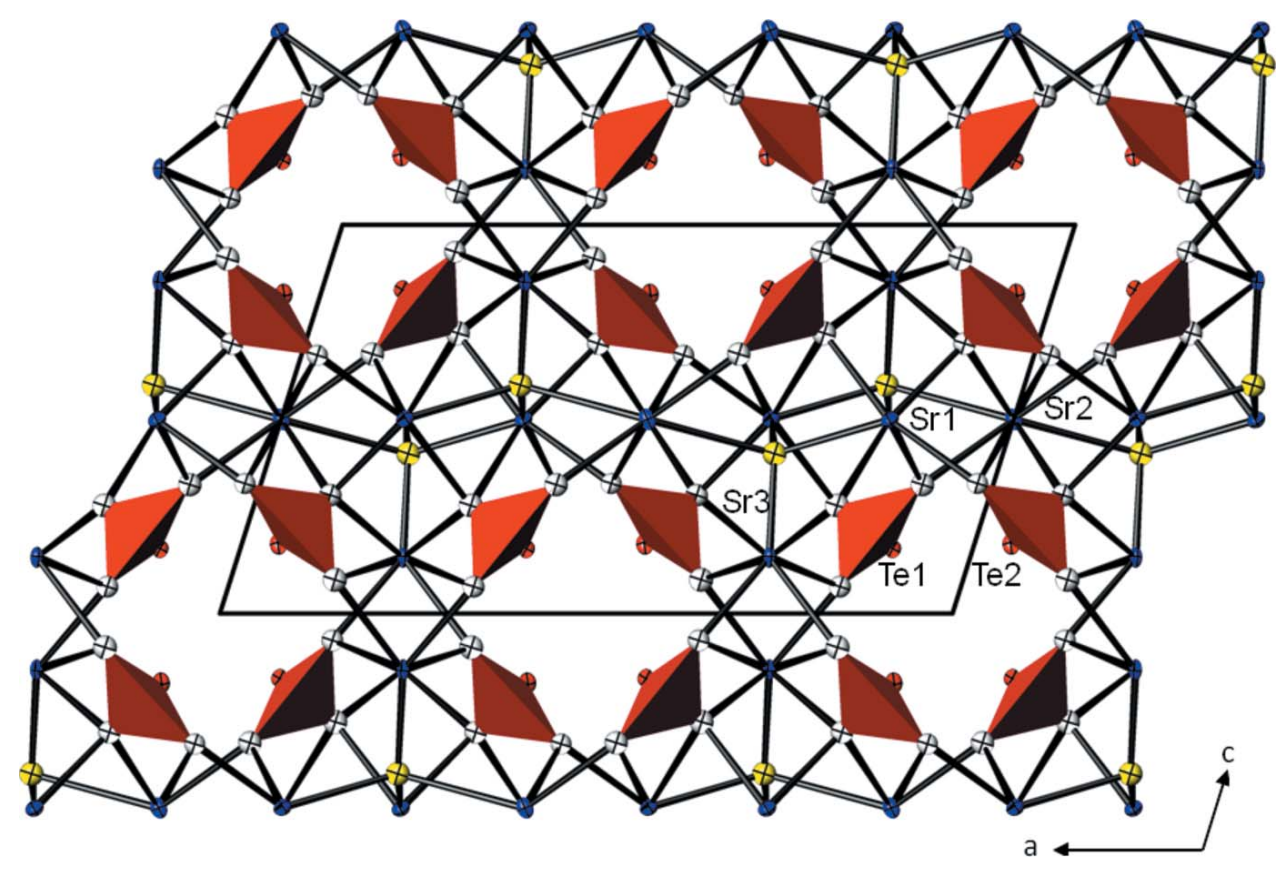

Figure 1

Projection of the crystal structure of $\mathrm{Sr}_{5} \mathrm{Te}_{4} \mathrm{O}_{12}(\mathrm{OH})_{2}$ along [010], with displacement ellipsoids drawn at the 74\% probability level. The trigonalpyramidal $\mathrm{TeO}_{3}$ groups are given in red; the $\mathrm{O}$ atom representing the $\mathrm{OH}$ group is given in yellow, all other $\mathrm{O}$ atoms are colourless. 
Table 3

Hydrogen-bond geometry $(\AA)$.

\begin{tabular}{ll}
\hline$D-\mathrm{H} \cdots A$ & $D \cdots A$ \\
\hline $\mathrm{O} 7 \cdots \mathrm{O} 5$ & $2.808(12)$ \\
$\mathrm{O} 7 \cdots \mathrm{O} 2$ & $2.893(12)$ \\
$\mathrm{O} 7 \cdots \mathrm{O} 2^{\text {ix }}$ & $2.991(11)$ \\
$\mathrm{O}^{\mathrm{i}} \cdots 1^{\text {iv }}$ & $3.063(11)$ \\
\hline
\end{tabular}

Symmetry codes: (iv) $-x, y+1,-z+1$; (ix) $-x+\frac{1}{2}, y+\frac{1}{2},-z+1$.

Bond-valence calculations (Brown, 2002) clearly reveal the presence of an $\mathrm{OH}$ group for atom $\mathrm{O} 7$ (Table 2), also required by charge neutrality. Atom $\mathrm{O} 7$ is bonded to four $\mathrm{Sr}$ atoms (Table 1, Fig. 1) and has also four possible oxygen acceptor atoms for hydrogen bonding of medium to weak strength (Table 3). The situation of four possible acceptor atoms is displayed in Fig. 2 and makes it appear likely that the corresponding $\mathrm{H}$ atom of the $\mathrm{OH}$ group is positionally disordered and thus could not be located during the present study.

In the sense of a crystal-chemically more detailed formula, the title compound may alternatively be formulated as $4 \mathrm{SrTeO}_{3} \cdot \mathrm{Sr}(\mathrm{OH})_{2}$ and represents the first basic strontium oxotellurate(IV), viz. with the presence of an $\mathrm{OH}$ functionality. In comparison with the other strontium oxotellurates(IV) compiled in Section 3, all $\mathrm{Sr}-\mathrm{O}$ and $\mathrm{Te}-\mathrm{O}$ lengths are in similar ranges.

\section{Database survey}

In the Inorganic Crystal Structure Database (ICSD, 2016) structural data for the following hydrous or anhydrous

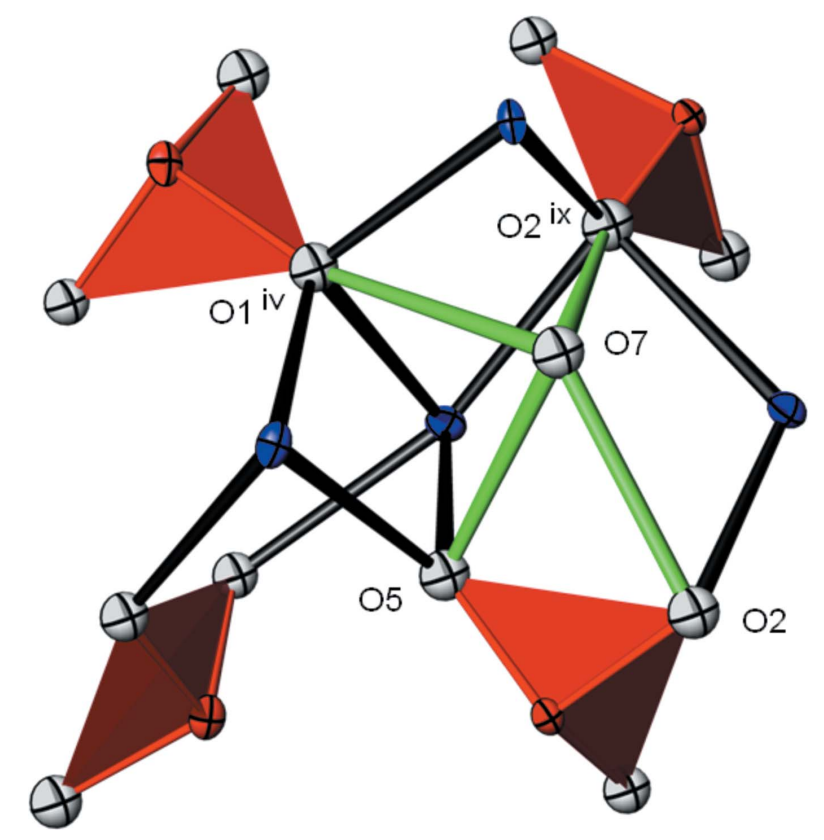

Figure 2

The vicinity of the $\mathrm{OH}$ group emphasizing the different possibilities for $\mathrm{O} \cdots \mathrm{O}$ hydrogen bonding (green lines). $\mathrm{Sr}-(\mathrm{OH})$ bonds have been omitted for clarity. Symmetry operators refer to those of Table 3; displacement ellipsoids are the same as in Fig. 1.
Table 4

Experimental details.

\begin{tabular}{|c|c|}
\hline \multicolumn{2}{|l|}{ Crystal data } \\
\hline Chemical formula & $\mathrm{Sr}_{5} \mathrm{Te}_{4} \mathrm{O}_{12}(\mathrm{OH})_{2}$ \\
\hline$M_{\mathrm{r}}$ & 1174.52 \\
\hline Crystal system, space group & Monoclinic, $C 2$ \\
\hline Temperature $(\mathrm{K})$ & 295 \\
\hline$a, b, c(\AA)$ & $16.0785(10), 5.7927(5), 8.9262(7)$ \\
\hline$\beta\left(^{\circ}\right)$ & $107.542(4)$ \\
\hline$V\left(\AA^{3}\right)$ & $792.71(11)$ \\
\hline$Z$ & 2 \\
\hline Radiation type & Мо $K \alpha$ \\
\hline$\mu\left(\mathrm{mm}^{-1}\right)$ & 23.99 \\
\hline Crystal size $(\mathrm{mm})$ & $0.18 \times 0.06 \times 0.01$ \\
\hline \multicolumn{2}{|l|}{ Data collection } \\
\hline Diffractometer & Bruker APEXII CCD \\
\hline Absorption correction & $\begin{array}{l}\text { Multi-scan (SADABS; Bruker, } \\
\text { 2012) }\end{array}$ \\
\hline$T_{\min }, T_{\max }$ & $0.099,0.795$ \\
\hline $\begin{array}{l}\text { No. of measured, independent and } \\
\text { observed }[I>2 \sigma(I)] \text { reflections }\end{array}$ & 12913, 1914, 1319 \\
\hline$R_{\text {int }}$ & 0.088 \\
\hline$(\sin \theta / \lambda)_{\max }\left(\AA^{-1}\right)$ & 0.660 \\
\hline \multicolumn{2}{|l|}{ Refinement } \\
\hline$R\left[F^{2}>2 \sigma\left(F^{2}\right)\right], w R\left(F^{2}\right), S$ & $0.042,0.085,1.02$ \\
\hline No. of reflections & 1914 \\
\hline No. of parameters & 71 \\
\hline No. of restraints & 1 \\
\hline $\mathrm{H}$-atom treatment & H-atom parameters not defined \\
\hline$\Delta \rho_{\max }, \Delta \rho_{\min }\left(\mathrm{e} \AA^{-3}\right)$ & $2.31,-1.78$ \\
\hline Absolute structure & Refined as an inversion twin \\
\hline Absolute structure parameter & $0.058(18)$ \\
\hline
\end{tabular}

Computer programs: APEX2 and SAINT (Bruker, 2012), SHELXS97 (Sheldrick, 2008), SHELXL2014 (Sheldrick, 2015), ATOMS (Dowty, 2006) and publCIF (Westrip, 2010).

strontium oxotellurate(IV) phases have been deposited: $\mathrm{SrTe}_{5} \mathrm{O}_{11}$ (Burckhardt \& Trömel, 1983), $\mathrm{Sr}_{3} \mathrm{Te}_{4} \mathrm{O}_{11}$ (Dytyatyev \& Dolgikh, 1999), various polymorphs of $\mathrm{SrTeO}_{3}$ (Dityatiev et al., 2006; Zavodnik et al., 2007a,b,c, 2008; Stöger et al., 2011), $\mathrm{SrTe}_{3} \mathrm{O}_{8}$ (Barrier et al., 2006; Weil \& Stöger, 2007) and $\mathrm{SrTeO}_{3}\left(\mathrm{H}_{2} \mathrm{O}\right)$ (Stöger et al., 2011). Additionally, in the International Centre for Diffraction Data PDF-4 database (ICDD, 2015) diffraction data for the following phases are compiled: $\mathrm{Sr}_{2} \mathrm{Te}_{3} \mathrm{O}_{8}$ (Elerman \& Koçak, 1986), $\mathrm{SrTe}_{2} \mathrm{O}_{5}$ (Redman et al., 1970; Gorbenko et al., 1983) and a high-temperature phase of the latter (Külcü et al., 1984).

\section{Synthesis and crystallization}

For the hydrothermal experiment, a Teflon container was filled with $0.0733 \mathrm{~g}$ of strontium oxide, $0.1529 \mathrm{~g}$ of tellurium dioxide and $0.032 \mathrm{ml}$ of selenic acid (conc.; $96 \mathrm{wt} \%$ ), corresponding to the stoichiometric ratio 3:2:1. To this mixture $10 \mathrm{ml}$ water were added to about three-fourth of the container volume. The container was then sealed with a Teflon lid and loaded into a stainless steel autoclave and then heated at autogenous pressure in an oven at $403 \mathrm{~K}$ for one week. After the reaction time, the autoclave was allowed to cool down to room temperature over six $\mathrm{h}$. The formed solid product was filtered off and washed with water and ethanol. Inspection under a polarizing microscope revealed a phase mixture with different 
crystal forms clearly discernible. According to X-ray powder diffraction of the bulk material, the following phases could be identified: $\alpha-\mathrm{TeO}_{2}$ (Lindqvist, 1968), $\mathrm{SrTe}_{2} \mathrm{O}_{5}$ (Redman et al., 1970), $\mathrm{SrTe}_{3} \mathrm{O}_{8}$ (Barrier et al., 2006; Weil \& Stöger, 2007) and $\mathrm{SrTe}_{5} \mathrm{O}_{11}$ (Burckhardt \& Trömel, 1983). Solid reaction products containing Se-phases were not detected. Platy $\mathrm{Sr}_{5} \mathrm{Te}_{4} \mathrm{O}_{12}(\mathrm{OH})_{2}$ crystals were present in only minor amounts, and were manually separated for structure determination from the other solid products.

\section{Refinement}

Crystal data, data collection and structure refinement details are summarized in Table 4. Some of the $\mathrm{O}$ atoms showed physically unreasonable behaviour when refined with anisotropic displacement parameters. Hence, for the final model all $\mathrm{O}$ atoms were refined with individual isotropic displacement parameters. The $\mathrm{H}$ atom of the $\mathrm{OH}$ group (or positionally disordered parts) could not be located and thus was not included in the model. Twinning by inversion was also taken into account, with a contribution of the minor twin component of about $6 \%$. The maximum and minimum remaining electron densities are found 2.34 and $0.96 \AA$, respectively, from $\mathrm{Sr} 3$.

\section{Acknowledgements}

The X-ray centre of the Vienna University of Technology is acknowledged for providing access to the single-crystal diffractometer.

\section{References}

Barrier, N., Malo, S., Hernandez, O., Hervieu, M. \& Raveau, B. (2006). J. Solid State Chem. 179, 3484-3488.

Brown, I. D. (2002). In The Chemical Bond in Inorganic Chemistry: The Bond Valence Model. Oxford University Press.

Brown, I. D. \& Altermatt, D. (1985). Acta Cryst. B41, 244-247.
Bruker (2012). APEX2, SAINT and SADABS. Bruker AXS Inc., Madison, Wisconsin, USA.

Burckhardt, H.-G. \& Trömel, M. (1983). Acta Cryst. C39, 1322-1323.

Christy, A. G., Mills, S. J. \& Kampf, A. R. (2016). Mineral. Mag. 80, 415-545.

Dityatiev, O. A., Berdonosov, P. S., Dolgikh, V. A., Aldous, D. W. \& Lightfoot, P. (2006). Solid State Sci. 8, 830-835.

Dowty, E. (2006). ATOMS for Windows. Shape Software, Kingsport, Tennessee, USA.

Dytyatyev, O. A. \& Dolgikh, V. A. (1999). Mater. Res. Bull. 34, 733740.

Elerman, Y. \& Koçak, M. (1986). J. Appl. Cryst. 19, 410.

Gorbenko, V. M., Kudzin, A. Yu. \& Sadovskaya, L. Ya. (1983). Inorg. Mater. (Engl. Transl.), 19, 267-300.

ICDD (2015). PDF-4+ 2015 Database, edited by S. Kabekkodu. International Centre for Diffraction Data, Newtown Square, PA, USA.

ICSD (2016). Inorganic Crystal Structure Database. FIZ-Karlsruhe, Germany. http://www.fiz-karlsruhe. de/icsd. html

Külcü, N., Burckhardt, H. G. \& Trömel, M. (1984). J. Solid State Chem. 2, 243-244.

Lindqvist, O. (1968). Acta Chem. Scand. 22, 977-982.

Redman, M. J., Chen, J. H., Binnie, W. P. \& Mallio, W. J. (1970). J. Am. Ceram. Soc. 53, 645-648.

Sheldrick, G. M. (2008). Acta Cryst. A64, 112-122.

Sheldrick, G. M. (2015). Acta Cryst. C71, 3-8.

Stöger, B. \& Weil, M. (2013). Miner. Petrol. 107, 253-263.

Stöger, B., Weil, M., Baran, E. J., González-Baró, A. C., Malo, S., Rueff, J. M., Petit, S., Lepetit, M. B., Raveau, B. \& Barrier, N. (2011). Dalton Trans. 40, 5538-5548.

Weil, M. (2015). Acta Cryst. C71, 712-716.

Weil, M. \& Shirkanlou, M. (2015). Z. Anorg. Allg. Chem. 641, 14591466.

Weil, M. \& Stöger, B. (2007). Acta Cryst. E63, i116-i118.

Westrip, S. P. (2010). J. Appl. Cryst. 43, 920-925.

Zavodnik, V. E., Ivanov, S. A. \& Stash, A. I. (2007a). Acta Cryst. E63, i75-i76.

Zavodnik, V. E., Ivanov, S. A. \& Stash, A. I. (2007b). Acta Cryst. E63, i111-i112.

Zavodnik, V. E., Ivanov, S. A. \& Stash, A. I. (2007c). Acta Cryst. E63, i151.

Zavodnik, V. E., Ivanov, S. A. \& Stash, A. I. (2008). Acta Cryst. E63, i52. 


\section{supporting information}

Acta Cryst. (2016). E72, 1532-1535 [https://doi.org/10.1107/S2056989016015577]

\section{Crystal structure of $\mathrm{Sr}_{5} \mathrm{Te}_{4} \mathrm{O}_{12}(\mathrm{OH})_{2}$, the first basic strontium oxotellurate(IV)}

\section{Matthias Weil and Mahdi Shirkhanlou}

\section{Computing details}

Data collection: APEX2 (Bruker, 2012); cell refinement: SAINT (Bruker, 2012); data reduction: SAINT (Bruker, 2012); program(s) used to solve structure: SHELXS97 (Sheldrick, 2008); program(s) used to refine structure: SHELXL2014 (Sheldrick, 2015); molecular graphics: ATOMS (Dowty, 2006); software used to prepare material for publication: publCIF (Westrip, 2010).

Pentastrontium tetrakis[oxotellurate(IV)] dihydroxide

Crystal data

$\mathrm{Sr}_{5} \mathrm{Te}_{4} \mathrm{O}_{12}(\mathrm{OH})_{2}$

$M_{r}=1174.52$

Monoclinic, $C 2$

Hall symbol: $\mathrm{C} 2 \mathrm{y}$

$a=16.0785$ (10) $\AA$

$b=5.7927(5) \AA$

$c=8.9262(7) \AA$

$\beta=107.542(4)^{\circ}$

$V=792.71(11) \AA^{3}$

$Z=2$

$$
F(000)=1024
$$

$D_{\mathrm{x}}=4.921 \mathrm{Mg} \mathrm{m}^{-3}$

Mo $K \alpha$ radiation, $\lambda=0.71073 \AA$

Cell parameters from 2028 reflections

$\theta=4.2-33.0^{\circ}$

$\mu=23.99 \mathrm{~mm}^{-1}$

$T=295 \mathrm{~K}$

Plate, colourless

$0.18 \times 0.06 \times 0.01 \mathrm{~mm}$

\section{Data collection}

Bruker APEXII CCD

diffractometer

Radiation source: fine-focus sealed tube

$\omega$ and $\varphi$ scans

Absorption correction: multi-scan

(SADABS; Bruker, 2012)

$T_{\min }=0.099, T_{\max }=0.795$

12913 measured reflections

1914 independent reflections 1319 reflections with $I>2 \sigma(I)$

$R_{\text {int }}=0.088$

$\theta_{\text {max }}=28.0^{\circ}, \theta_{\text {min }}=2.4^{\circ}$

$h=-20 \rightarrow 21$

$k=-7 \rightarrow 7$

$l=-11 \rightarrow 11$

$\mathrm{H}$-atom parameters not defined

$w=1 /\left[\sigma^{2}\left(F_{\mathrm{o}}{ }^{2}\right)+(0.0236 P)^{2}+0.7139 P\right]$

where $P=\left(F_{\mathrm{o}}{ }^{2}+2 F_{\mathrm{c}}{ }^{2}\right) / 3$

$(\Delta / \sigma)_{\max }<0.001$

$\Delta \rho_{\max }=2.31 \mathrm{e}^{-3}$

$\Delta \rho_{\min }=-1.78$ e $\AA^{-3}$

Absolute structure: Refined as an inversion twin

Absolute structure parameter: 0.058 (18) 


\section{Special details}

Geometry. All esds (except the esd in the dihedral angle between two 1.s. planes) are estimated using the full covariance matrix. The cell esds are taken into account individually in the estimation of esds in distances, angles and torsion angles; correlations between esds in cell parameters are only used when they are defined by crystal symmetry. An approximate (isotropic) treatment of cell esds is used for estimating esds involving l.s. planes.

Refinement. Refined as a 2-component inversion twin

Fractional atomic coordinates and isotropic or equivalent isotropic displacement parameters $\left(\AA^{2}\right)$

\begin{tabular}{lllll}
\hline & $x$ & $y$ & $z$ & $U_{\text {iso }} * / U_{\text {eq }}$ \\
\hline Sr1 & $0.16911(10)$ & $0.0360(4)$ & $0.4988(2)$ & $0.0097(5)$ \\
Sr2 & 0.0000 & $0.5471(4)$ & 0.5000 & $0.0094(8)$ \\
Sr3 & $0.27399(11)$ & $0.0613(2)$ & $0.1447(2)$ & $0.0071(5)$ \\
Te1 & $0.10820(7)$ & $0.52512(19)$ & $0.16455(15)$ & $0.0081(3)$ \\
Te2 & $-0.05011(7)$ & $0.0246(2)$ & $0.17800(14)$ & $0.0091(3)$ \\
O1 & $-0.0998(7)$ & $-0.1557(19)$ & $0.3050(14)$ & $0.015(3)^{*}$ \\
O2 & $0.2040(8)$ & $0.3491(18)$ & $0.2785(14)$ & $0.017(3)^{*}$ \\
O3 & $0.0218(7)$ & $0.2152(19)$ & $0.3350(14)$ & $0.013(3)^{*}$ \\
O4 & $-0.1423(7)$ & $0.2229(19)$ & $0.0869(14)$ & $0.018(3)^{*}$ \\
O5 & $0.0964(7)$ & $0.7088(19)$ & $0.3320(14)$ & $0.017(3)^{*}$ \\
O6 & $0.1651(7)$ & $0.7327(19)$ & $0.0690(14)$ & $0.014(3)^{*}$ \\
O7 & $0.1881(7)$ & $0.4343(18)$ & $0.5881(13)$ & $0.017(3)^{*}$ \\
\hline
\end{tabular}

Atomic displacement parameters $\left(\AA^{2}\right)$

\begin{tabular}{lllllll}
\hline & $U^{11}$ & $U^{22}$ & $U^{33}$ & $U^{12}$ & $U^{13}$ & $U^{23}$ \\
\hline Sr1 & $0.0090(8)$ & $0.0107(11)$ & $0.0086(10)$ & $-0.0015(8)$ & $0.0013(7)$ & $-0.0017(9)$ \\
Sr2 & $0.0108(13)$ & $0.006(2)$ & $0.0134(14)$ & 0.000 & $0.0063(10)$ & 0.000 \\
Sr3 & $0.0055(8)$ & $0.0035(13)$ & $0.0127(10)$ & $-0.0001(7)$ & $0.0036(6)$ & $-0.0012(9)$ \\
Te1 & $0.0066(6)$ & $0.0084(8)$ & $0.0087(6)$ & $0.0003(6)$ & $0.0014(4)$ & $0.0000(6)$ \\
Te2 & $0.0092(6)$ & $0.0062(7)$ & $0.0126(7)$ & $-0.0013(7)$ & $0.0041(4)$ & $-0.0024(8)$ \\
\hline
\end{tabular}

Geometric parameters $\left(\AA,{ }^{\circ}\right)$

\begin{tabular}{llll}
\hline $\mathrm{Sr} 1-\mathrm{O} 7$ & $2.430(12)$ & $\mathrm{Sr}-\mathrm{O} 2$ & $2.507(11)$ \\
$\mathrm{Sr} 1-\mathrm{O} 5^{\mathrm{i}}$ & $2.476(12)$ & $\mathrm{Sr}-\mathrm{O} 4^{\mathrm{vi}}$ & $2.517(11)$ \\
$\mathrm{Sr} 1-\mathrm{O} 1^{\mathrm{ii}}$ & $2.593(12)$ & $\mathrm{Sr} 3-\mathrm{O} 6^{\mathrm{i}}$ & $2.536(11)$ \\
$\mathrm{Sr} 1-\mathrm{O} 3$ & $2.596(11)$ & $\mathrm{Sr} 3-\mathrm{O}^{\mathrm{vii}}$ & $2.590(12)$ \\
$\mathrm{Sr} 1-\mathrm{O} 2^{\mathrm{iii}}$ & $2.616(12)$ & $\mathrm{Sr} 3-\mathrm{O} 4^{\mathrm{viii}}$ & $2.644(11)$ \\
$\mathrm{Sr} 1-\mathrm{O} 7^{\mathrm{iii}}$ & $2.700(11)$ & $\mathrm{Sr} 3-\mathrm{O} 1^{\mathrm{ix}}$ & $2.666(11)$ \\
$\mathrm{Sr} 1-\mathrm{O} 2$ & $2.852(12)$ & $\mathrm{Te} 1-\mathrm{O} 6$ & $1.865(11)$ \\
$\mathrm{Sr}-\mathrm{O} 3$ & $2.510(11)$ & $\mathrm{Te} 1-\mathrm{O} 2$ & $1.871(11)$ \\
$\mathrm{Sr} 2-\mathrm{O} 3^{\mathrm{ii}}$ & $2.510(11)$ & $\mathrm{Te} 1-\mathrm{O} 5$ & $1.850(12)$ \\
$\mathrm{Sr} 2-\mathrm{O} 1^{\mathrm{iv}}$ & $2.624(12)$ & $\mathrm{Te} 2-\mathrm{O} 4$ & $1.882(11)$ \\
$\mathrm{Sr} 2-\mathrm{O} 1^{\mathrm{v}}$ & $2.624(11)$ & $\mathrm{Te} 2-\mathrm{O} 3$ & $1.886(11)$ \\
$\mathrm{Sr} 2-\mathrm{O} 5$ & $2.633(12)$ & $\mathrm{Te} 2-\mathrm{O} 1$ & $3.063(11)$ \\
$\mathrm{Sr} 2-\mathrm{O} 5^{\mathrm{ii}}$ & $2.633(12)$ & $\mathrm{O} 7-\mathrm{O} 1^{\mathrm{ii}}$ & $2.893(12)$ \\
$\mathrm{Sr} 2-\mathrm{O} 7^{\mathrm{ii}}$ & $2.960(11)$ & $\mathrm{O} 7-\mathrm{O} 2$ &
\end{tabular}


$\mathrm{Sr} 2-\mathrm{O} 7$

$\mathrm{Sr} 3-\mathrm{O} 7^{\mathrm{iii}}$

$\mathrm{O} 7-\mathrm{Sr} 1-\mathrm{O} 5^{\mathrm{i}}$

$\mathrm{O} 7-\mathrm{Sr} 1-\mathrm{O}^{\mathrm{ii}}$

$\mathrm{O} 5^{\mathrm{i}}-\mathrm{Sr} 1-\mathrm{O} 1^{\mathrm{ii}}$

$\mathrm{O} 7-\mathrm{Sr} 1-\mathrm{O} 3$

$\mathrm{O} 5$ - $-\mathrm{Sr} 1-\mathrm{O} 3$

$\mathrm{O} 1$ ii $-\mathrm{Sr} 1-\mathrm{O} 3$

$\mathrm{O} 7-\mathrm{Sr} 1-\mathrm{O} 2^{\mathrm{iii}}$

$\mathrm{O} 5^{\mathrm{i}}-\mathrm{Sr} 1-\mathrm{O} 2^{\mathrm{iii}}$

$\mathrm{O} 1^{\mathrm{ii}}-\mathrm{Sr} 1-\mathrm{O} 2^{\mathrm{iii}}$

$\mathrm{O} 3-\mathrm{Sr} 1-\mathrm{O} 2^{\mathrm{iii}}$

$\mathrm{O} 7-\mathrm{Sr} 1-\mathrm{O} 7^{\mathrm{iii}}$

$\mathrm{O} 5$ - $\mathrm{Sr} 1-\mathrm{O} 7^{\mathrm{iii}}$

$\mathrm{O} 1^{\mathrm{ii}}-\mathrm{Sr} 1-\mathrm{O} 7^{\mathrm{iii}}$

$\mathrm{O} 3-\mathrm{Sr} 1-\mathrm{O} 7^{\mathrm{iii}}$

$\mathrm{O} 2^{\mathrm{iii}}-\mathrm{Sr} 1-\mathrm{O} 7^{\mathrm{iii}}$

$\mathrm{O} 7-\mathrm{Sr} 1-\mathrm{O} 2$

$\mathrm{O} 5$ - $-\mathrm{Sr} 1-\mathrm{O} 2$

$\mathrm{O} 1 \mathrm{ii}-\mathrm{Sr} 1-\mathrm{O} 2$

$\mathrm{O} 3-\mathrm{Sr} 1-\mathrm{O} 2$

$\mathrm{O} 2{ }^{\mathrm{iii}}-\mathrm{Sr} 1-\mathrm{O} 2$

$\mathrm{O} 7$ iii- $\mathrm{Sr} 1-\mathrm{O} 2$

$\mathrm{O} 3-\mathrm{Sr} 2-\mathrm{O} 3^{\mathrm{ii}}$

$\mathrm{O} 3-\mathrm{Sr} 2-\mathrm{O} 1^{\text {iv }}$

$\mathrm{O}^{3 i}-\mathrm{Sr} 2-\mathrm{O}^{\mathrm{iv}}$

$\mathrm{O} 3-\mathrm{Sr} 2-\mathrm{O}^{\mathrm{v}}$

$\mathrm{O} 3{ }^{\mathrm{ii}}-\mathrm{Sr} 2-\mathrm{O}^{\mathrm{v}}$

$\mathrm{O} 1^{\mathrm{iv}}-\mathrm{Sr} 2-\mathrm{O} 1^{\mathrm{v}}$

$\mathrm{O} 3-\mathrm{Sr} 2-\mathrm{O} 5$

$\mathrm{O} 3{ }^{\mathrm{ii}}-\mathrm{Sr} 2-\mathrm{O} 5$

$\mathrm{O} 1^{\mathrm{iv}}-\mathrm{Sr} 2-\mathrm{O} 5$

$\mathrm{O} 1$ - $-\mathrm{Sr} 2-\mathrm{O} 5$

$\mathrm{O} 3-\mathrm{Sr} 2-\mathrm{O} 5^{\mathrm{ii}}$

$\mathrm{O} 3^{\mathrm{ii}}-\mathrm{Sr} 2-\mathrm{O} 5^{\mathrm{ii}}$

$\mathrm{O} 1^{\mathrm{iv}}-\mathrm{Sr} 2-\mathrm{O}^{3 i}$

$\mathrm{O} 1^{\mathrm{v}}-\mathrm{Sr} 2-\mathrm{O} 5^{\mathrm{ii}}$

$\mathrm{O} 5-\mathrm{Sr} 2-\mathrm{O} 5^{\mathrm{ii}}$

$\mathrm{O} 3-\mathrm{Sr} 2-\mathrm{O}^{\mathrm{ii}}$

$\mathrm{O} 3^{\mathrm{ii}}-\mathrm{Sr} 2-\mathrm{O} 7^{\mathrm{ii}}$

$\mathrm{O} 1^{\mathrm{iv}}-\mathrm{Sr} 2-\mathrm{O} 7^{\mathrm{ii}}$

$\mathrm{O} 1^{\mathrm{v}}-\mathrm{Sr} 2-\mathrm{O} 7^{\mathrm{ii}}$

$\mathrm{O} 5-\mathrm{Sr} 2-\mathrm{O} 7^{\mathrm{ii}}$

$\mathrm{O} 5^{\mathrm{ii}}-\mathrm{Sr} 2-\mathrm{O} 7^{\mathrm{ii}}$

O3- $\mathrm{Sr} 2-\mathrm{O} 7$

$\mathrm{O} 3$ ii- $\mathrm{Sr} 2-\mathrm{O} 7$

$\mathrm{O} 1^{\mathrm{iv}}-\mathrm{Sr} 2-\mathrm{O} 7$
$2.960(11)$

2.393 (11)

$156.1(4)$

$102.8(4)$

81.8 (4)

79.0 (4)

77.4 (4)

92.7 (4)

98.7 (4)

$105.0(4)$

72.8 (4)

164.5 (4)

$105.5(3)$

87.0 (4)

$132.6(4)$

$129.5(4)$

65.9 (4)

65.9 (4)

$103.2(4)$

$162.2(4)$

72.1 (3)

121.1 (2)

$65.2(3)$

$80.0(5)$

$136.6(4)$

$106.2(3)$

$106.2(3)$

$136.6(4)$

$98.0(5)$

74.2 (3)

$144.8(4)$

78.3 (4)

74.7 (3)

144.8 (4)

74.2 (3)

74.7 (3)

78.3 (4)

$138.3(5)$

89.3 (3)

71.0 (3)

133.8 (3)

66.2 (3)

$131.1(3)$

60.0 (3)

71.0 (3)

89.3 (3)

$66.2(3)$
$\mathrm{O} 7-\mathrm{O} 2^{\mathrm{x}}$

O7-O5

2.991 (11)

2.808 (12)

104.7 (4)

74.3 (3)

144.4 (4)

114.8 (4)

78.7 (4)

118.4 (3)

142.3 (4)

76.6 (4)

117.8 (3)

74.4 (3)

$71.3(3)$

74.3 (4)

73.4 (4)

102.8 (3)

163.2 (4)

76.3 (3)

$120.2(3)$

99.4 (4)

100.3 (5)

98.0 (5)

$101.1(5)$

$100.3(5)$

98.8 (5)

$121.0(5)$

118.5 (5)

97.7 (4)

$114.2(5)$

102.3 (4)

99.6 (4)

$121.2(6)$

120.9 (5)

106.2 (4)

114.9 (5)

96.6 (3)

90.5 (4)

$136.3(5)$

115.9 (5)

103.9 (4)

$142.8(6)$

$117.9(5)$

94.8 (4)

139.9 (6)

117.8 (5)

100.5 (4)

139.1 (6) 


$\begin{array}{llll}\mathrm{O}^{\mathrm{v}}-\mathrm{Sr} 2-\mathrm{O} 7 & 133.8(3) & \mathrm{Te} 1-\mathrm{O} 6-\mathrm{Sr} 3^{\mathrm{xiii}} & 115.9(5) \\ \mathrm{O} 5-\mathrm{Sr} 2-\mathrm{O} 7 & 60.0(3) & \mathrm{Sr} 3^{\mathrm{v}}-\mathrm{O} 6-\mathrm{Sr} 3^{\mathrm{xiii}} & 95.7(4) \\ \mathrm{O} 5^{\mathrm{ii}-\mathrm{Sr} 2-\mathrm{O} 7} & 131.1(3) & \mathrm{Sr} 3^{\mathrm{x}}-\mathrm{O} 7-\mathrm{Sr} 1 & 126.0(5) \\ \mathrm{O} 7^{\mathrm{ii}-\mathrm{Sr} 2-\mathrm{O} 7} & 154.5(4) & \mathrm{Sr} 3^{\mathrm{x}}-\mathrm{O} 7-\mathrm{Sr}^{\mathrm{x}} & 103.7(4) \\ \mathrm{O} 7^{\mathrm{ii}}-\mathrm{Sr} 3-\mathrm{O} 2 & 75.2(4) & \mathrm{Sr} 1-\mathrm{O} 7-\mathrm{Sr} 1^{\mathrm{x}} & 98.5(4) \\ \mathrm{O} 7^{\mathrm{iii}}-\mathrm{Sr} 3-\mathrm{O} 4^{\mathrm{vi}} & 88.5(4) & \mathrm{Sr} 3^{\mathrm{x}}-\mathrm{O} 7-\mathrm{Sr} 2 & 96.4(4) \\ \mathrm{O} 2-\mathrm{Sr} 3-\mathrm{O} 4^{\mathrm{vi}} & 163.7(4) & \mathrm{Sr} 1-\mathrm{O} 7-\mathrm{Sr} 2 & 139.9(4) \\ \mathrm{O} 7^{\mathrm{iii}}-\mathrm{Sr} 3-6^{\mathrm{i}} & 89.0(4) & \mathrm{Sr} 1^{\mathrm{x}}-\mathrm{O} 7-\mathrm{Sr} 2 & \end{array}$

Symmetry codes: (i) $x, y-1, z$; (ii) $-x, y,-z+1$; (iii) $-x+1 / 2, y-1 / 2,-z+1$; (iv) $-x, y+1,-z+1$; (v) $x, y+1, z$; (vi) $x+1 / 2, y-1 / 2, z$; (vii) $-x+1 / 2, y-1 / 2,-z$; (viii) $-x, y,-z$; (ix) $x+1 / 2, y+1 / 2, z$; (x) $-x+1 / 2, y+1 / 2,-z+1$; (xi) $x-1 / 2, y-1 / 2, z$; (xii) $x-1 / 2, y+1 / 2, z$; (xiii) $-x+1 / 2, y+1 / 2,-z$.

Hydrogen-bond geometry (A)

\begin{tabular}{ll}
\hline$D-\mathrm{H} \cdots A$ & $D \cdots A$ \\
\hline $\mathrm{O} 7 \cdots \mathrm{O} 5$ & $2.808(12)$ \\
$\mathrm{O} 7 \cdots \mathrm{O} 2$ & $2.893(12)$ \\
$\mathrm{O} 7 \cdots \mathrm{O} 2^{\mathrm{x}}$ & $2.991(11)$ \\
$\mathrm{O} 7 \cdots \mathrm{O} 1^{\text {iv }}$ & $3.063(11)$ \\
\hline
\end{tabular}

Symmetry codes: (iv) $-x, y+1,-z+1$; (x) $-x+1 / 2, y+1 / 2,-z+1$. 